\title{
On the Henstock-Kurzweil integral for Riesz-space-valued functions on time scales
}

\author{
Xuexiao You ${ }^{\mathrm{a}, \mathrm{b}}$, Dafang Zhao ${ }^{\mathrm{a}, \mathrm{c}}$, Delfim F. M. Torres ${ }^{\mathrm{d}, *}$ \\ a School of Mathematics and Statistics, Hubei Normal University, Huangshi, Hubei 435002, P. R. China. \\ ${ }^{b}$ College of Computer and Information, Hohai University, Nanjing, Jiangsu 210098, P. R. China. \\ ${ }^{c}$ College of Science, Hohai University, Nanjing, Jiangsu 210098, P. R. China. \\ ${ }^{d}$ Center for Research and Development in Mathematics and Applications (CIDMA), Department of Mathematics, University of Aveiro, \\ 3810-193 Aveiro, Portugal.
}

Communicated by A. Atangana

\begin{abstract}
We introduce and investigate the Henstock-Kurzweil (HK) integral for Riesz-space-valued functions on time scales. Some basic properties of the HK delta integral for Riesz-space-valued functions are proved. Further, we prove uniform and monotone convergence theorems. (C)2017 All rights reserved.
\end{abstract}

Keywords: Henstock-Kurzweil integral, Riesz space, time scales. 2010 MSC: 28B05, 28B10, 28B15, 46G10.

\section{Introduction}

It is well-known that the Henstock-Kurzweil integral integrates highly oscillating functions and encompasses Newton, Riemann and Lebesgue integrals. This integral was introduced by Kurzweil and Henstock independently in 1957/58 [34, 39]. It has been shown that the Henstock-Kurzweil integral is equivalent to the Denjoy-Perron integral. For fundamental results and some applications in the theory of Henstock-Kurzweil integration, we refer the reader to the papers [23-25, 27, 32-34, 60, 61, 64, 66] and monographs $[4,30,35,36,38,40-45,48,55,56]$.

A time scale $\mathbb{T}$ is an arbitrary nonempty closed subset of real numbers $\mathbb{R}$ with the subspace topology inherited from the standard topology of $\mathbb{R}$. The theory of time scales was born in 1988 with the Ph.D. thesis of Hilger [37]. The aim of this theory is to unify various definitions and results from the theories of discrete and continuous dynamical systems, and to extend such theories to more general classes of dynamical systems. It has been extensively studied on various aspects by several authors; see, e.g., [5, 6, 19-22, 31, 46]. In [47], Peterson and Thompson introduced a more general concept of integral on time scales, i.e., the Henstock-Kurzweil delta integral, which contains the Riemann delta and the Lebesguedelta integrals as special cases. The theory of Henstock-Kurzweil integration for real-valued

\footnotetext{
${ }^{*}$ Corresponding author

Email addresses: youxuexiao@126.com (Xuexiao You), dafangzhao@163.com (Dafang Zhao), delfim@ua.pt (Delfim F. M. Torres)

doi:10.22436/jnsa.010.05.18
} 
and vector-valued functions on time scales has developed rather intensively in the past few years; see, for instance, the papers $[1-3,26,28,54,57-59,62,65]$ and the references cited therein.

One of the interesting points of integration theories is the problem when functions with values in general spaces have to be integrated. The Henstock-Kurzweil integral for Riesz-space-valued functions was investigated in [7-18, 49-53, 63]. Surprisingly enough, the Henstock-Kurzweil integral for Rieszspace-valued functions has not received attention in the literature of time scales. The main goal of this paper is to generalize the results above by constructing the Henstock-Kurzweil integral for Riesz-spacevalued functions on time scales.

The paper is organized as follows. Section 2 contains basic concepts of Riesz space, time scales and Henstock-Kurzweil integral. In Section 3, the definition of Henstock-Kurzweil delta integral for Rieszspace-valued functions is introduced, and the basic properties of the Henstock-Kurzweil delta integral for Riesz-space-valued functions are investigated. In Section 4, we prove a uniformly convergence theorem and a monotone convergence theorem for the Henstock-Kurzweil delta integral for Riesz-space-valued functions.

\section{Preliminaries}

The following conventions and notations will be used, unless stated otherwise. Let $\mathbb{N}, \mathbb{R}$, and $\mathbb{R}^{+}$ be the sets of all natural, real, and positive real numbers, respectively, and let $X$ be a Riesz space. A decreasing sequence $\left(b_{n}\right)_{n}$ in $X$, such that $\bigwedge_{n} b_{n}=0$, is called an (o)-sequence. A bounded double sequence $\left(a_{i, j}\right)_{i, j}$ in $X$ is a (D)-sequence or a regulator if $\left(a_{i, j}\right)_{i, j}$ is an (o)-sequence for all $i \in \mathbb{N}$. A Riesz space $X$ is said to be Dedekind complete if every nonempty subset $X_{1}$ of $X$, bounded from above, has a lattice supremum in $X$ denoted by $\bigvee X_{1}$. A Dedekind complete Riesz space $X$ is said to be weakly $\sigma$-distributive if for every (D)-sequence $\left(a_{i, j}\right)_{i, j}$ in $X$ one has:

$$
\bigwedge_{\varphi \in \mathbb{N}^{\mathbb{N}}}\left(\bigvee_{i=1}^{\infty} a_{i, \varphi(i)}\right)=0 .
$$

Let $\mathbb{T}$ be a time scale, i.e., a nonempty closed subset of $\mathbb{R}$. For $a, b \in \mathbb{T}$, we define the closed interval $[a, b]_{\mathbb{T}}$ by $[a, b]_{\mathbb{T}}=\{t \in \mathbb{T}: a \leqslant t \leqslant b\}$. The open and half-open intervals are defined in a similar way. For $t \in \mathbb{T}$, we define the forward jump operator $\sigma$ by $\sigma(t)=\inf \{s>t: s \in \mathbb{T}\}$, where $\inf \emptyset=\sup \{\mathbb{T}\}$, while the backward jump operator $\rho$ is defined by $\rho(t)=\sup \{s<t: s \in \mathbb{T}\}$, where $\sup \emptyset=\inf \{\mathbb{T}\}$.

If $\sigma(t)>t$, then we say that $t$ is right-scattered, while if $\rho(t)<t$, then we say that $t$ is left-scattered. If $\sigma(t)=t$, then we say that $t$ is right-dense, while if $\rho(t)=t$, then we say that $t$ is left-dense. A point $t \in \mathbb{T}$ is dense if it is right and left dense; isolated, if it is right and left scattered. The forward graininess function $\mu(t)$ and the backward graininess function $\eta(t)$ are defined by $\mu(t)=\sigma(t)-t$ and $\eta(t)=t-\rho(t)$ for all $t \in \mathbb{T}$, respectively. If sup $\mathbb{T}$ is finite and left-scattered, then we define $\mathbb{T}^{k}:=\mathbb{T} \backslash \sup \mathbb{T}$, otherwise $\mathbb{T}^{k}:=\mathbb{T}$; if inf $\mathbb{T}$ is finite and right-scattered, then $\mathbb{T}_{K}:=\mathbb{T} \backslash \inf \mathbb{T}$, otherwise $\mathbb{T}_{K}:=\mathbb{T}$. We set $\mathbb{T}_{K}^{K}:=\mathbb{T}^{K} \cap \mathbb{T}_{k}$.

Throughout this paper, all considered intervals will be intervals in $\mathbb{T}$. A partition $\mathcal{D}$ of $[a, b]_{\mathbb{T}}$ is a finite collection of interval-point pairs $\left\{\left(\left[t_{i-1}, t_{i}\right]_{\mathbb{T}}, \xi_{i}\right)\right\}_{i=1}^{n}$, where

$$
\left\{a=t_{0}<t_{1}<\cdots<t_{n-1}<t_{n}=b\right\}
$$

and $\xi_{i} \in[a, b]_{\mathbb{T}}$ for $i=1,2, \cdots, n$. By $\Delta t_{i}=t_{i}-t_{i-1}$ we denote the length of the $i$ th subinterval in the partition $\mathcal{D}$. We say that $\delta(\xi)=\left(\delta_{\mathrm{L}}(\xi), \delta_{\mathrm{R}}(\xi)\right)$ is $\Delta$-gauge for $[a, b]_{\mathbb{T}}$ provided $\delta_{\mathrm{L}}(\xi)>0$ on $(a, b]_{\mathbb{T}}$, $\delta_{R}(\xi)>0$ on $[a, b)_{\mathbb{T}}, \delta_{L}(a) \geqslant 0, \delta_{R}(b) \geqslant 0$ and $\delta_{R}(\xi) \geqslant \mu(\xi)$ for all $\xi \in[a, b)_{\mathbb{T}}$. Let $\delta^{1}(\xi), \delta^{2}(\xi)$ be $\Delta$-gauges for $[a, b]_{\mathbb{T}}$ such that $0<\delta_{\mathrm{L}}^{1}(\xi)<\delta_{\mathrm{L}}^{2}(\xi)$ for all $\xi \in(\mathrm{a}, \mathrm{b}]_{\mathbb{T}}$ and $0<\delta_{\mathrm{R}}^{1}(\xi)<\delta_{\mathrm{R}}^{2}(\xi)$ for all $\xi \in[\mathrm{a}, \mathrm{b})_{\mathbb{T}}$. We say $\delta^{1}(\xi)$ is finer than $\delta^{2}(\xi)$ and write $\delta^{1}(\xi)<\delta^{2}(\xi)$. We say that $\mathcal{D}=\left\{\left(\left[t_{i-1}, t_{i}\right]_{\mathbb{T}}, \xi_{i}\right)\right\}_{i=1}^{n}$ is

(1) a partial partition of $[a, b]_{\mathbb{T}}$ if $\bigcup_{i=1}^{n}\left[t_{i-1}, t_{i}\right]_{\mathbb{T}} \subset[a, b]_{\mathbb{T}}$;

(2) a partition of $[a, b]_{\mathbb{T}}$ if $\bigcup_{i=1}^{n}\left[t_{i-1}, t_{i}\right]_{\mathbb{T}}=[a, b]_{\mathbb{T}}$; 
(3) a $\delta$-fine Henstock-Kurzweil (HK) partition of $[a, b]_{\mathbb{T}}$ if $\xi_{i} \in\left[t_{i-1}, t_{i}\right]_{\mathbb{T}} \subset\left(\xi_{i}-\delta_{L}\left(\xi_{i}\right), \xi_{i}+\delta_{R}\left(\xi_{i}\right)\right)_{\mathbb{T}}$ for all $i=1,2, \ldots, n$.

Given a $\delta$-fine $H K$ partition $\mathcal{D}=\left\{\left(\left[t_{i-1}, t_{i}\right]_{\mathbb{T}}, \xi_{i}\right)\right\}_{i=1}^{n}$ of $[a, b]_{\mathbb{T}}$, we write

$$
S(f, \mathcal{D}, \delta)=\sum_{i=1}^{n} f\left(\xi_{i}\right)\left(t_{i}-t_{i-1}\right)
$$

for integral sums over $D$, whenever $f:[a, b]_{\mathbb{T}} \rightarrow X$. In what follows, we shall always assume that $X$ is a Dedekind complete weakly $\sigma$-distributive Riesz space.

\section{The Henstock-Kurzweil delta integral for Riesz-space-valued functions}

Before formulating and giving the proof of our first result, we need the following definition.

Definition 3.1. A function $f:[a, b]_{\mathbb{T}} \rightarrow X$ is called Henstock-Kurzweil delta integrable (HK $\Delta$-integrable) on $[a, b]_{\mathbb{T}}$, if there exists $x \in X$ and a $(D)$-sequence $\left(a_{i, j}\right)_{i, j}$ of elements of $X$ such that for every $\varphi \in \mathbb{N}^{\mathbb{N}}$ there exists a $\Delta$-gauge, $\delta$, for $[a, b]_{\mathbb{T}}$, such that

$$
|S(f, \mathcal{D}, \delta)-x|<\bigvee_{i=1}^{\infty} a_{i, \varphi(i)}
$$

for each $\delta$-fine HK partition $\mathcal{D}=\left\{\left(\left[t_{i-1}, t_{i}\right]_{\mathbb{T}}, \xi_{i}\right)\right\}_{i=1}^{n}$ of $[a, b]_{\mathbb{T}}$. In this case, $x$ is called the HK $\Delta$-integral of $f$ on $[a, b]_{\mathbb{T}}$ and is denoted by $x=\int_{a}^{b} f(t) \Delta t$.

Theorem 3.2. If $\mathrm{f}:[\mathrm{a}, \mathrm{b}]_{\mathbb{T}} \rightarrow \mathrm{X}$ is HK $\Delta$-integrable on $[\mathrm{a}, \mathrm{b}]_{\mathbb{T}}$, then the integral of $\mathrm{f}$ is determined uniquely.

Proof. Suppose there exist $x_{1}, x_{2} \in X$ and (D)-sequences $\left(a_{i, j}\right)_{i, j},\left(b_{i, j}\right)_{i, j}$ of elements of $X$ and for every $\varphi \in \mathbb{N}^{\mathbb{N}}$ there exist two $\Delta$-gauges, $\delta_{1}, \delta_{2}$, for $[a, b]_{\mathbb{T}}$, such that

$$
\left|S\left(f, \mathcal{D}_{1}, \delta_{1}\right)-x_{1}\right|<\bigvee_{i=1}^{\infty} a_{i, \varphi(i)}, \quad\left|S\left(f, \mathcal{D}_{2}, \delta_{2}\right)-x_{2}\right|<\bigvee_{i=1}^{\infty} b_{i, \varphi(i)}
$$

for each $\delta_{1}$-fine HK partition $\mathcal{D}_{1}$ and $\delta_{2}$-fine HK partition $\mathcal{D}_{2}$, respectively. Let $\delta=\min \left\{\delta_{1}, \delta_{2}\right\}$ and $\left(c_{i, j}\right)_{i, j}$ be a (D)-sequence of elements of $X$ such that

$$
\bigvee_{i=1}^{\infty} a_{i, \varphi(i)}+\bigvee_{i=1}^{\infty} b_{i, \varphi(i)} \leqslant \bigvee_{i=1}^{\infty} c_{i, \varphi(i)}
$$

for every $\varphi \in \mathbb{N}^{\mathbb{N}}$. Then,

$$
\left|x_{1}-x_{2}\right| \leqslant\left|S(f, \mathcal{D}, \delta)-x_{1}\right|+\left|S(f, \mathcal{D}, \delta)-x_{2}\right|<\bigvee_{i=1}^{\infty} a_{i, \varphi(i)}+\bigvee_{i=1}^{\infty} b_{i, \varphi(i)} \leqslant \bigvee_{i=1}^{\infty} c_{i, \varphi(i)}
$$

for each $\delta$-fine $H K$ partition $\mathcal{D}$. Since $X$ is weakly $\sigma$-distributive, we obtain that

$$
\left|x_{1}-x_{2}\right| \leqslant \bigwedge_{\varphi \in \mathbb{N}^{\mathbb{N}}}\left(\bigvee_{i=1}^{\infty} c_{i, \varphi(i)}\right)=0 \text {. }
$$

The proof is complete.

Theorem 3.3. If $\mathrm{f}, \mathrm{g}:[\mathrm{a}, \mathrm{b}]_{\mathbb{T}} \rightarrow \mathrm{X}$ are $H K \Delta$-integrable on $[\mathrm{a}, \mathrm{b}]_{\mathbb{T}}$ and $\alpha, \beta \in \mathbb{R}$, then $\alpha \mathrm{f}+\beta \mathrm{g}$ is $H K \Delta$-integrable on $[a, b]_{\mathbb{T}}$ and

$$
\int_{a}^{b}(\alpha f(t)+\beta g(t)) \Delta t=\alpha \int_{a}^{b} f(t) \Delta t+\beta \int_{a}^{b} g(t) \Delta t .
$$


Proof. We shall prove that if $f, g$ are $H K \Delta$-integrable on $[a, b]_{\mathbb{T}}$ and $c \in \mathbb{R}$, then $f+g$ and cf are HK $\Delta$-integrable too and

$$
\int_{a}^{b}(f(t)+g(t)) \Delta t=\int_{a}^{b} f(t) \Delta t+\int_{a}^{b} g(t) \Delta t, \quad \int_{a}^{b} c f(t) \Delta t=c \int_{a}^{b} f(t) \Delta t
$$

If $f$ is HK $\Delta$-integrable on $[a, b]_{\mathbb{T}}$, then there exists a $(D)$-sequence $\left(a_{i, j}\right)_{i, j}$ of elements of $X$ such that for every $\varphi \in \mathbb{N}^{\mathbb{N}}$ there exists a $\Delta$-gauge, $\delta_{1}$, for $[a, b]_{\mathbb{T}}$, such that

$$
\left|S\left(f, \mathcal{D}_{1}, \delta_{1}\right)-\int_{a}^{b} f(t) \Delta t\right|<\bigvee_{i=1}^{\infty} a_{i, \varphi(i)}
$$

for each $\delta_{1}$-fine HK partition $\mathcal{D}_{1}$. Similarly, there exists a (D)-sequence $\left(b_{i, j}\right)_{i, j}$ of elements of $X$ such that for every $\varphi \in \mathbb{N}^{\mathbb{N}}$ there exists a $\Delta$-gauge, $\delta_{2}$, for $[a, b]_{\mathbb{T}}$, such that

$$
\left|S\left(g, \mathcal{D}_{2}, \delta_{2}\right)-\int_{a}^{b} g(t) \Delta t\right|<\bigvee_{i=1}^{\infty} b_{i, \varphi(i)}
$$

for each $\delta_{2}$-fine HK partition $\mathcal{D}_{2}$. Let $\delta=\min \left\{\delta_{1}, \delta_{2}\right\}$, and consider a $(D)$-sequence $\left(c_{i, j}\right)_{i, j}$ of elements of $X$ such that

$$
\bigvee_{i=1}^{\infty} a_{i, \varphi(i)}+\bigvee_{i=1}^{\infty} b_{i, \varphi(i)} \leqslant \bigvee_{i=1}^{\infty} c_{i, \varphi(i)}
$$

for every $\varphi \in \mathbb{N}^{\mathbb{N}}$. Then,

$$
\begin{aligned}
\left|S(f+g, \mathcal{D}, \delta)-\int_{a}^{b} f(t) \Delta t-\int_{a}^{b} g(t) \Delta t\right| & =\left|S(f, \mathcal{D}, \delta)-\int_{a}^{b} f(t) \Delta t+S(g, \mathcal{D}, \delta)-\int_{a}^{b} g(t) \Delta t\right| \\
& \leqslant\left|S(f, \mathcal{D}, \delta)-\int_{a}^{b} f(t) \Delta t\right|+\left|S(g, \mathcal{D}, \delta)-\int_{a}^{b} g(t) \Delta t\right| \\
& <\bigvee_{i=1}^{\infty} a_{i, \varphi(i)}+\bigvee_{i=1}^{\infty} b_{i, \varphi(i)} \leqslant \bigvee_{i=1}^{\infty} c_{i, \varphi(i)}
\end{aligned}
$$

for each $\delta$-fine $H K$ partition $\mathcal{D}$. Hence, $f+g$ is $H K \Delta$-integrable and

$$
\int_{a}^{b}(f(t)+g(t)) \Delta t=\int_{a}^{b} f(t) \Delta t+\int_{a}^{b} g(t) \Delta t
$$

For $c \in \mathbb{R},\left(|c| a_{i, j}\right)_{i, j}$ is a (D)-sequence. Then,

$$
\left|S(c f, \mathcal{D}, \delta)-c \int_{a}^{b} f(t) \Delta t\right| \leqslant|c|\left|S(f, \mathcal{D}, \delta)-\int_{a}^{b} f(t) \Delta t\right|<|c| \bigvee_{i=1}^{\infty} a_{i, \varphi(i)}=\bigvee_{i=1}^{\infty}|c| a_{i, \varphi(i)}
$$

for each $\delta$-fine $H K$ partition $\mathcal{D}$. This implies that $\mathrm{cf}$ is $\mathrm{HK} \Delta$-integrable and

$$
\int_{a}^{b} c f(t) \Delta t=c \int_{a}^{b} f(t) \Delta t
$$

The proof is complete.

Theorem 3.4 (Cauchy-Bolzano condition). A function $\mathrm{f}:[\mathrm{a}, \mathrm{b}]_{\mathbb{T}} \rightarrow \mathrm{X}$ is $H K \Delta$-integrable on $[\mathrm{a}, \mathrm{b}]_{\mathbb{T}}$ if and only if there exists a (D)-sequence $\left(a_{i, j}\right)_{i, j}$ of elements of $\mathrm{X}$ such that for every $\varphi \in \mathbb{N}^{\mathbb{N}}$ there exists a $\Delta$-gauge, $\delta$, for $[\mathrm{a}, \mathrm{b}]_{\mathbb{T}}$, such that

$$
\left|S\left(f, \mathcal{D}_{1}, \delta\right)-S\left(f, \mathcal{D}_{2}, \delta\right)\right|<\bigvee_{i=1}^{\infty} a_{i, \varphi(i)}
$$

for each $\delta$-fine $H K$ partition $\mathcal{D}_{1}, \mathcal{D}_{2}$ of $[a, b]_{\mathbb{T}}$. 
Proof.

(Necessity). This follows from the inequality

$$
\left|S\left(f, \mathcal{D}_{1}, \delta\right)-S\left(f, \mathcal{D}_{2}, \delta\right)\right| \leqslant\left|S\left(f, \mathcal{D}_{1}, \delta\right)-\int_{a}^{b} f(t) \Delta t\right|+\left|S\left(f, \mathcal{D}_{2}, \delta\right)-\int_{a}^{b} f(t) \Delta t\right|
$$

and some routine arguments.

(Sufficiency). To every $\varphi \in \mathbb{N}^{\mathbb{N}}$, there exists a $\Delta$-gauge $\delta_{\varphi}(\xi)$ with the following property. Let

$$
\delta_{[a, b]_{\mathbb{T}}}=\left\{\delta(\xi): \exists \varphi \in \mathbb{N}^{\mathbb{N}}, \delta(\xi)=\delta_{\varphi}(\xi), \xi \in[a, b]_{\mathbb{T}}\right\} .
$$

Then, for $\delta(\xi) \in \delta_{[a, b]_{\mathbb{T}}}$ and a $\delta$-fine HK partition $\mathcal{D}$, the set $\{S(f, \mathcal{D}, \delta)\}$ is bounded. Indeed, for $X$ boundedly complete, there exist

$$
a_{\delta}=\bigwedge_{\mathcal{D}} S(f, \mathcal{D}, \delta), \quad b_{\delta}=\bigvee_{\mathcal{D}} S(f, \mathcal{D}, \delta) .
$$

For $\delta_{1}(\xi), \delta_{2}(\xi) \in \delta_{[a, b]_{\mathbb{T}}}$ let $\delta(\xi)=\min \left\{\delta_{1}(\xi), \delta_{2}(\xi)\right\}$. Then,

$$
a_{\delta_{1}}=\bigwedge_{\mathcal{D}} S\left(f, \mathcal{D}, \delta_{1}\right) \leqslant \bigwedge_{\mathcal{D}} S(f, \mathcal{D}, \delta) \leqslant \bigvee_{\mathcal{D}} S(f, \mathcal{D}, \delta) \leqslant \bigvee_{\mathcal{D}} S\left(f, \mathcal{D}, \delta_{2}\right)=b_{\delta_{2}}
$$

Therefore,

$$
\bigvee_{\delta(\xi) \in \delta_{[a, b]_{T}}} a_{\delta} \leqslant \bigwedge_{\delta(\xi) \in \delta_{[a, b]_{T}}} b_{\delta}
$$

Hence, there exists $x \in X$ such that $a_{\delta} \leqslant x \leqslant b_{\delta}$ for all $\delta(\xi) \in \delta_{[a, b]_{\mathbb{T}}}$. Now, let $\varphi \in \mathbb{N}^{\mathbb{N}}$. Then there exists a $\Delta$-gauge $\delta_{\varphi}(\xi)$ for $[a, b]_{\mathbb{T}}$ such that

$$
S\left(f, \mathcal{D}_{1}, \delta_{\varphi}\right) \leqslant S\left(f, \mathcal{D}_{2}, \delta_{\varphi}\right)+\bigvee_{i=1}^{\infty} a_{i, \varphi(i)}
$$

for each $\delta_{\varphi}$-fine $\mathrm{HK}$ partition $\mathcal{D}_{1}, \mathcal{D}_{2}$. Fix $\mathcal{D}_{2}$. Then

$$
b_{\delta_{\varphi}} \leqslant S\left(f, \mathcal{D}_{2}, \delta_{\varphi}\right)+\bigvee_{i=1}^{\infty} a_{i, \varphi(i)} .
$$

Since the inequality holds for every $\delta_{\varphi}$-fine $\mathrm{HK}$ partition $\mathcal{D}_{2}$, we have

$$
b_{\delta_{\varphi}} \leqslant a_{\delta_{\varphi}}+\bigvee_{i=1}^{\infty} a_{i, \varphi(i)} .
$$

By the weak $\sigma$-distributivity of $X$, we obtain that

$$
\bigwedge_{\varphi \in \mathbb{N}^{\mathbb{N}}}\left(\bigvee_{i=1}^{\infty} a_{i, \varphi(i)}\right)=0
$$

and so

$$
\bigwedge_{\varphi \in \mathbb{N}^{\mathbb{N}}} b_{\delta_{\varphi}}-\bigvee_{\varphi \in \mathbb{N}^{\mathbb{N}}} a_{\delta_{\varphi}} \leqslant \bigwedge_{\varphi \in \mathbb{N}^{\mathbb{N}}}\left(b_{\delta_{\varphi}}-a_{\delta_{\varphi}}\right)=0
$$

Consequently,

$$
x=\bigwedge_{\varphi \in \mathbb{N}^{\mathbb{N}}} b_{\delta_{\varphi}}=\bigvee_{\varphi \in \mathbb{N}^{\mathbb{N}}} a_{\delta_{\varphi}}
$$


Then, for every $\delta_{\varphi}$-fine $H K$ partition $\mathcal{D}$, we have

$$
S\left(f, \mathcal{D}, \delta_{\varphi}\right)-x \leqslant b_{\delta_{\varphi}}-a_{\delta_{\varphi}} \leqslant \bigvee_{i=1}^{\infty} a_{i, \varphi(i)}, \quad x-S\left(f, \mathcal{D}, \delta_{\varphi}\right) \leqslant b_{\delta_{\varphi}}-a_{\delta_{\varphi}} \leqslant \bigvee_{i=1}^{\infty} a_{i, \varphi(i)} .
$$

It follows that

$$
\left|S\left(f, \mathcal{D}, \delta_{\varphi}\right)-x\right| \leqslant \bigvee_{i=1}^{\infty} a_{i, \varphi(i)}
$$

and the proof is complete.

Theorem 3.5. If $\mathrm{f}:[\mathrm{a}, \mathrm{b}]_{\mathbb{T}} \rightarrow \mathrm{X}$, then $\mathrm{f}$ is $H K-\Delta$ integrable on $[\mathrm{a}, \mathrm{b}]_{\mathbb{T}}$ if and only if $\mathrm{f}$ is $H K-\Delta$ integrable on $[\mathrm{a}, \mathrm{c}]_{\mathbb{T}}$ and $[\mathrm{c}, \mathrm{b}]_{\mathbb{T}}$. Moreover, in this case

$$
\int_{a}^{b} f(t) \Delta t=\int_{a}^{c} f(t) \Delta t+\int_{c}^{b} f(t) \Delta t
$$

Proof.

(Necessity). By Theorem 3.4, there exists a (D)-sequence $\left(a_{i, j}\right)_{i, j}$ of elements of $X$ such that for every $\varphi \in \mathbb{N}^{\mathbb{N}}$, there exists a $\Delta$-gauge, $\delta$, for $[a, b]_{\mathbb{T}}$, such that

$$
\left|S\left(f, \mathcal{D}_{1}, \delta\right)-S\left(f, \mathcal{D}_{2}, \delta\right)\right|<\bigvee_{i=1}^{\infty} a_{i, \varphi(i)}
$$

for each $\delta$-fine $H K$ partition $\mathcal{D}_{1}, \mathcal{D}_{2}$ of $[a, b]_{\mathbb{T}}$. Take any two $\delta$-fine $H K$ partitions of $[a, c]_{\mathbb{T}}$, say, $\mathcal{D}_{3}$ and $\mathcal{D}_{4}$. Similarly, take another $\delta$-fine HK partition $\mathcal{D}_{5}$ of $[c, b]_{\mathbb{T}}$. Then, we have

$$
\left|S\left(f, \mathcal{D}_{3}, \delta\right)-S\left(f, \mathcal{D}_{4}, \delta\right)\right|=\left|S\left(f, \mathcal{D}_{3}+\mathcal{D}_{5}, \delta\right)-S\left(f, \mathcal{D}_{5}, \delta\right)+S\left(f, \mathcal{D}_{5}, \delta\right)-S\left(f, \mathcal{D}_{4}+\mathcal{D}_{5}, \delta\right)\right|<\bigvee_{i=1}^{\infty} a_{i, \varphi(i)}
$$

Hence, $f$ is HK- $\Delta$ integrable on $[a, c]_{\mathbb{T}}$. Similarly, $f$ is HK- $\Delta$ integrable on $[c, b]_{\mathbb{T}}$. Consequently, there exist (D)-sequences $\left(a_{i, j}\right)_{i, j},\left(b_{i, j}\right)_{i, j}$ and $\left(c_{i, j}\right)_{i, j}$ of elements of $X$ such that for every $\varphi \in \mathbb{N}^{\mathbb{N}}$ there exists a $\Delta$-gauge, $\delta$, for $[a, b]_{\mathbb{T}}$, such that

$$
\begin{aligned}
\left|S(f, \mathcal{D}, \delta)-\int_{a}^{b} f(t) \Delta t\right| & <\bigvee_{i=1}^{\infty} a_{i, \varphi(i)},\left|S\left(f, \mathcal{D}^{\prime}, \delta\right)-\int_{a}^{c} f(t) \Delta t\right|<\bigvee_{i=1}^{\infty} b_{i, \varphi(i)}, \\
\left|S\left(f, \mathcal{D}-\mathcal{D}^{\prime}, \delta\right)-\int_{c}^{b} f(t) \Delta t\right| & <\bigvee_{i=1}^{\infty} c_{i, \varphi(i)}
\end{aligned}
$$

for each $\delta$-fine $H K$ partition $\mathcal{D}$ of $[a, b]_{\mathbb{T}}, \mathcal{D}^{\prime}$, of $[a, c]_{\mathbb{T}}$ and $\mathcal{D}-\mathcal{D}^{\prime}$ of $[c, b]_{\mathbb{T}}$. Then, there exists a (D)sequence $\left(d_{i, j}\right)_{i, j}$ of elements of $X$ such that

$$
\begin{aligned}
\mid \int_{a}^{b} f(t) \Delta t & -\int_{a}^{c} f(t) \Delta t-\int_{c}^{b} f(t) \Delta t \mid \\
& \leqslant\left|S(f, \mathcal{D}, \delta)-\int_{a}^{b} f(t) \Delta t\right|+\left|S\left(f, \mathcal{D}^{\prime}, \delta\right)-\int_{a}^{c} f(t) \Delta t\right|+\left|S\left(f, \mathcal{D}-\mathcal{D}^{\prime}, \delta\right)-\int_{c}^{b} f(t) \Delta t\right| \\
& \leqslant \bigvee_{i=1}^{\infty} a_{i, \varphi(i)}+\bigvee_{i=1}^{\infty} b_{i, \varphi(i)}+\bigvee_{i=1}^{\infty} c_{i, \varphi(i)} \leqslant \bigvee_{i=1}^{\infty} d_{i, \varphi(i)}
\end{aligned}
$$

and the result follows. 
(Sufficiency). Let $f$ be HK- $\Delta$ integrable on $[a, c]_{\mathbb{T}}$ and $[c, b]_{\mathbb{T}}$. Then there exist (D)-sequences $\left(a_{i, j}\right)_{i, j}$, $\left(b_{i, j}\right)_{i, j}$ of elements of $X$ such that for every $\varphi \in \mathbb{N}^{\mathbb{N}}$ there exist $\Delta$-gauges,

$$
\delta^{1}(\xi)=\left(\delta_{\mathrm{L}}^{1}(\xi), \delta_{\mathrm{R}}^{1}(\xi)\right), \quad \delta^{2}(\xi)=\left(\delta_{\mathrm{L}}^{2}(\xi), \delta_{\mathrm{R}}^{2}(\xi)\right),
$$

for $[a, b]_{\mathbb{T}}$ such that

$$
\left|S\left(f, \mathcal{D}_{1}, \delta^{1}\right)-\int_{a}^{c} f(t) \Delta t\right|<\bigvee_{i=1}^{\infty} a_{i, \varphi(i)}, \quad\left|S\left(f, \mathcal{D}_{2}, \delta^{2}\right)-\int_{c}^{b} f(t) \Delta t\right|<\bigvee_{i=1}^{\infty} b_{i, \varphi(i)}
$$

for each $\delta^{1}$-fine HK partition $\mathcal{D}_{1}=\left\{\left(\left[t_{k-1}^{1}, t_{k}^{1}\right]_{\mathbb{T}}, \xi_{k}^{1}\right)\right\}_{k=1}^{n}$ of $[a, c]_{\mathbb{T}}$ and for each $\delta^{2}$-fine HK partition $\mathcal{D}_{2}=\left\{\left(\left[t_{k-1}^{2}, t_{k}^{2}\right]_{\mathbb{T}}, \xi_{k}^{2}\right)\right\}_{k=1}^{m}$ of $[c, b]_{\mathbb{T}}$, respectively. We define a $\Delta$-gauge $\delta(\xi)=\left(\delta_{L}(\xi), \delta_{R}(\xi)\right)$, on $[a, b]_{\mathbb{T}}$, by first defining $\delta_{\mathrm{L}}(\xi)$ as

$$
\delta_{\mathrm{L}}(\xi)= \begin{cases}\delta_{\mathrm{L}}^{1}(\xi), & \text { if } \xi \in[a, c)_{\mathbb{T}}, \\ \delta_{\mathrm{L}}^{1}(\xi), & \text { if } \xi=c=\rho(\mathrm{c}), \\ \min \left\{\delta_{\mathrm{L}}^{1}(\xi), \frac{\eta(c)}{2}\right\}, & \text { if } \xi=c>\rho(\mathrm{c}), \\ \min \left\{\delta_{\mathrm{L}}^{2}(\xi), \frac{\xi-c}{2}\right\}, & \text { if } \xi \in(c, b]_{\mathbb{T}},\end{cases}
$$

and then defining $\delta_{R}(\xi)$ as

$$
\delta_{R}(\xi)= \begin{cases}\min \left\{\delta_{R}^{1}(\xi), \max \left\{\mu(\xi), \frac{c-\xi}{2}\right\}\right\}, & \text { if } \xi \in[a, c)_{\mathbb{T}}, \\ \min \left\{\delta_{R}^{2}(\xi)\right\}, & \text { if } \xi \in[c, b]_{\mathbb{T}} .\end{cases}
$$

Now, let $\mathcal{D}=\left\{\left(\left[t_{k-1}, t_{k}\right]_{\mathbb{T}}, \xi_{k}\right)\right\}_{k=1}^{p}$ be a $\delta$-fine HK partition of $[a, b]_{\mathbb{T}}$. Then, either $c$ is a tag point for $\mathcal{D}$, say $c=\xi_{q}$, and $t_{q}>c$; or $\rho(c)<c$, and $\rho(c)$ is a tag point for $\mathcal{D}$, say $\rho(c)=\xi_{q}$, and $t_{q}=c$. In the first case, there exists a (D)-sequence $\left(c_{i, j}\right)_{i, j}$ of elements of $X$ such that for every $\varphi \in \mathbb{N}^{\mathbb{N}}$ we have

$$
\begin{aligned}
\left|S(f, \mathcal{D}, \delta)-\int_{a}^{c} f(t) \Delta t-\int_{c}^{b} f(t) \Delta t\right|= & \left|\sum_{k=1}^{p} f\left(\xi_{k}\right)\left(t_{k}-t_{k-1}\right)-\int_{a}^{c} f(t) \Delta t-\int_{c}^{b} f(t) \Delta t\right| \\
\leqslant & \left|\sum_{k=1}^{q-1} f\left(\xi_{k}\right)\left(t_{k}-t_{k-1}\right)+f(c)\left(c-t_{q-1}\right)-\int_{a}^{c} f(t) \Delta t\right| \\
& +\left|\sum_{k=q+1}^{p} f\left(\xi_{k}\right)\left(t_{k}-t_{k-1}\right)+f(c)\left(t_{q}-c\right)-\int_{c}^{b} f(t) \Delta t\right| \\
& <\bigvee_{i=1}^{\infty} a_{i, \varphi(i)}+\bigvee_{i=1}^{\infty} b_{i, \varphi(i)}<\bigvee_{i=1}^{\infty} c_{i, \varphi(i)}
\end{aligned}
$$

Using the weak $\sigma$-distributivity, we get the corresponding results. The other case is easy and is omitted. Hence, $f$ is HK- $\Delta$-integrable on $[a, b]_{\mathbb{T}}$ and

$$
\int_{a}^{b} f(t) \Delta t=\int_{a}^{c} f(t) \Delta t+\int_{c}^{b} f(t) \Delta t
$$

This concludes the proof.

Lemma 3.6 (The Saks-Henstock lemma). Let $\mathrm{f}:[\mathrm{a}, \mathrm{b}]_{\mathbb{T}} \rightarrow \mathrm{X}$ be HK- $\Delta$ integrable on $[\mathrm{a}, \mathrm{b}]_{\mathbb{T}}$. Then there exists $a$ (D)-sequence $\left(a_{i, j}\right)_{i, j}$ of elements of $X$ such that for every $\varphi \in \mathbb{N}^{\mathbb{N}}$ there exists a $\Delta$-gauge, $\delta$, for $[a, b]_{\mathbb{T}}$, such that

$$
\left|S(f, \mathcal{D}, \delta)-\int_{a}^{b} f(t) \Delta t\right|<\bigvee_{i=1}^{\infty} a_{i, \varphi(i)}
$$


for each $\delta$-fine $H K$ partition $\mathcal{D}$ of $[a, b]_{\mathbb{T}}$. In particular, if $\mathcal{D}^{\prime}=\left\{\left(\left[t_{k-1}, t_{k}\right]_{\mathbb{T}}, \xi_{k}\right)\right\}_{k=1}^{m}$ is an arbitrary $\delta$-fine partial HK partition of $[\mathrm{a}, \mathrm{b}]_{\mathbb{T}}$, then

$$
\left|S\left(f, \mathcal{D}^{\prime}, \delta\right)-\sum_{k=1}^{m} \int_{t_{k-1}}^{t_{k}} f(t) \Delta t\right| \leqslant \bigvee_{i=1}^{\infty} a_{i, \varphi(i)} .
$$

Proof. Assume $\mathcal{D}^{\prime}=\left\{\left(\left[t_{k-1}, t_{k}\right]_{\mathbb{T}}, \xi_{k}\right)\right\}_{k=1}^{m}$ is an arbitrary $\delta^{\prime}$-fine partial HK partition of $[a, b]_{\mathbb{T}}$. Then the complement $[a, b]_{\mathbb{T}} \backslash \bigcup_{k=1}^{m}\left[t_{k-1}, k_{i}\right]_{\mathbb{T}}$ can be expressed as a fine collection of closed subintervals and we denote

$$
[a, b]_{\mathbb{T}} \backslash \bigcup_{k=1}^{m}\left[t_{k-1}, t_{k}\right]_{\mathbb{T}}=\sum_{k=1}^{n}\left[t_{k-1}^{\prime}, t_{k}^{\prime}\right]_{\mathbb{T}} .
$$

From Theorem 3.5, we know that $\int_{t_{k-1}^{\prime}}^{t_{k}^{\prime}} f(t) \Delta t$ exists. Then, there exist (D)-sequences $\left(b_{k, i, j}\right)_{k, i, j}$ of elements of $X$ such that for every $\varphi \in \mathbb{N}^{\mathbb{N}}$ there exist $\Delta$-gauges $\delta_{1}, \delta_{2}, \ldots, \delta_{n}$ for $[a, b]_{\mathbb{T}}$, such that

$$
\left|S\left(f, \mathcal{D}_{k}, \delta_{k}\right)-\int_{t_{k-1}^{\prime}}^{t_{k}^{\prime}} f(t) \Delta t\right|<\bigvee_{i=1}^{\infty} b_{k, i, \varphi(i)}
$$

for each $\delta_{k}$-fine HK partition $\mathcal{D}_{k}$ of $\left[t_{k-1}, t_{k}\right]_{\mathbb{T}}$. Assume that $\delta \leqslant \delta^{\prime}, \delta_{1}, \delta_{2}, \ldots, \delta_{n}$. Let

$$
\mathcal{D}_{0}=\mathcal{D}^{\prime}+\mathcal{D}_{1}+\mathcal{D}_{2}+\cdots+\mathcal{D}_{n}
$$

Obviously, $\mathcal{D}_{0}$ is a $\delta$-fine HK partition of $[a, b]_{\mathbb{T}}$. Then, there exists a (D)-sequence $\left(a_{i, j}\right)_{i, j}$ of elements of $\mathrm{X}$ such that

$$
\left|S\left(f, \mathcal{D}_{0}, \delta\right)-\int_{a}^{b} f(t) \Delta t\right|=\left|S\left(f, \mathcal{D}^{\prime}, \delta\right)+\sum_{k=1}^{n} S\left(f, \mathcal{D}_{k}, \delta\right)-\int_{a}^{b} f(t) \Delta t\right|<\bigvee_{i=1}^{\infty} a_{i, \varphi(i)}
$$

for every $\varphi \in \mathbb{N}^{\mathbb{N}}$. Consequently, we obtain

$$
\begin{aligned}
\left|S\left(f, \mathcal{D}^{\prime}, \delta\right)-\sum_{k=1}^{m} \int_{t_{k-1}}^{t_{k}} f(t) \Delta t\right| & =\left|S\left(f, \mathcal{D}_{0}, \delta\right)-\sum_{k=1}^{n} S\left(f, \mathcal{D}_{k}, \delta\right)-\left(\int_{a}^{b} f(t) \Delta t-\sum_{k=1}^{n} \int_{t_{k-1}^{\prime}}^{t_{k}^{\prime}} f(t) \Delta t\right)\right| \\
& \leqslant\left|S\left(f, \mathcal{D}_{0}, \delta\right)-\int_{a}^{b} f(t) \Delta t\right|+\sum_{k=1}^{n}\left|S\left(f, \mathcal{D}_{k}, \delta\right)-\int_{t_{k-1}^{\prime}}^{t_{k}^{\prime}} f(t) \Delta t\right| \\
& <\bigvee_{i=1}^{\infty} a_{i, \varphi(i)}+\sum_{k=1}^{n} \bigvee_{i=1}^{\infty} b_{k, i, \varphi(i)} \\
& \leqslant \bigvee_{i=1}^{\infty} a_{i, \varphi(i)}+\sum_{k=1}^{n} \bigvee_{i=1}^{\infty} \sum_{k=1}^{n} b_{k, i, \varphi(i)} \leqslant \bigvee_{i=1}^{\infty} a_{i, \varphi(i)}+n \bigvee_{i=1}^{\infty} \sum_{k=1}^{n} b_{k, i, \varphi(i)} .
\end{aligned}
$$

Let $c_{i, j}=n \sum_{k=1}^{n} b_{k, i, \varphi(j)}$. Then, $\left(c_{i, j}\right)_{i, j}$ is a (D)-sequence and, for every $\varphi \in \mathbb{N}^{\mathbb{N}}$, we have

$$
\left|S\left(f, \mathcal{D}^{\prime}, \delta\right)-\sum_{k=1}^{m} \int_{t_{k-1}}^{t_{k}} f(t) \Delta t\right| \leqslant \bigvee_{i=1}^{\infty} a_{i, \varphi(i)} .
$$

The proof is complete.

\section{Convergence theorems}

In this section we prove two convergence theorems. We begin with the following two definitions.

Definition 4.1. We say that $f_{n} \rightarrow f$ converges with a common regulating sequence (w.c.r.s.) if there exists 
a (D)-sequence $\left(a_{i, j}\right)_{i, j}$ of elements of $X$ such that for every $\varphi \in \mathbb{N}^{\mathbb{N}}$ and every $t \in[a, b]_{\mathbb{T}}$ there exists $p=p(t)$ such that

$$
\left|f_{n}(t)-f(t)\right|<\bigvee_{i=1}^{\infty} a_{i, \varphi(i)}
$$

for any $n \geqslant p$.

Definition 4.2. We say that $\left\{f_{n}\right\}_{n=1}^{\infty}$ is uniformly HK $\Delta$-integrable on $[a, b]_{T}$ if each $f_{n}$ is HK $\Delta$-integrable on $[a, b]_{\mathbb{T}}$ and there exists a (D)-sequence $\left(a_{i, j}\right)_{i, j}$ of elements of $X$ such that for every $\varphi \in \mathbb{N}^{\mathbb{N}}$ there exists a $\Delta$-gauge $\delta$ for $[a, b]_{\mathbb{T}}$, such that

$$
\left|S\left(f_{n}, \mathcal{D}, \delta\right)-\int_{a}^{b} f_{n}(t) \Delta t\right|<\bigvee_{i=1}^{\infty} b_{i, \varphi(i)}
$$

for each $\delta$-fine $H K$ partition $\mathcal{D}$ of $[a, b]_{\mathbb{T}}$ and $n \in \mathbb{N}$.

For uniformly HK $\Delta$-integrable sequences of integrable functions, we have the following convergence theorem.

Theorem 4.3. Let $\left\{\mathrm{f}_{\mathrm{n}}\right\}_{\mathrm{n}=1}^{\infty}$ be a sequence of uniformly HK $\Delta$-integrable functions on $[\mathrm{a}, \mathrm{b}]_{\mathbb{T}}$ and assume that $\mathrm{f}_{\mathrm{n}} \rightarrow \mathrm{f}$ converges with a common regulating sequence. Then $\mathrm{f}$ is $H K \Delta$-integrable and

$$
\lim _{n \rightarrow \infty} \int_{a}^{b} f_{n}(t) \Delta t=\int_{a}^{b} f(t) \Delta t
$$

Proof. We will prove the theorem in two steps.

Step 1. By assumption, there exists a (D)-sequence $\left(b_{i, j}\right)_{i, j}$ of elements of $X$ such that for every $\varphi \in \mathbb{N}^{\mathbb{N}}$, there exist two $\Delta$-gauges $\delta_{1}$ and $\delta_{2}$ for $[a, b]_{\mathbb{T}}$, such that

$$
\left|S\left(f_{n}, \mathcal{D}_{1}, \delta_{1}\right)-\int_{a}^{b} f_{n}(t) \Delta t\right|<\bigvee_{i=1}^{\infty} b_{i, \varphi(i+1)}, \quad\left|S\left(f_{n}, \mathcal{D}_{2}, \delta_{2}\right)-\int_{a}^{b} f_{n}(t) \Delta t\right|<\bigvee_{i=1}^{\infty} b_{i, \varphi(i+2)}
$$

for each $\delta_{1}$-fine $\left(\delta_{2}\right.$-fine) HK partition $\mathcal{D}_{1}=\left\{\left[t_{i-1}, t_{i}\right], \xi_{i}\right\}_{i}\left(\mathcal{D}_{2}=\left\{\left[t_{i-1}^{\prime}, t_{i}^{\prime}\right], \xi_{i}^{\prime}\right\}_{i}\right)$ of $[a, b]_{\mathbb{T}}$ and $n \in \mathbb{N}$. Let $\delta=\min \left\{\delta_{1}, \delta_{2}\right\}$. By the w.c.r.s. convergence,

$$
\left|S\left(f, \mathcal{D}_{1}, \delta\right)-S\left(f_{n}, \mathcal{D}_{1}, \delta\right)\right| \leqslant \sum_{i}\left|f\left(\xi_{i}\right)-f_{n}\left(\xi_{i}\right)\right|\left(t_{i}-t_{i-1}\right)<(b-a) \bigvee_{i=1}^{\infty} a_{i, \varphi(i+1)}
$$

for each $\delta$-fine HK partition $\mathcal{D}_{1}$ and $n \geqslant p_{1}$. Similarly, we have

$$
\left|S\left(f, \mathcal{D}_{2}, \delta\right)-S\left(f_{n}, \mathcal{D}_{2}, \delta\right)\right| \leqslant \sum_{i}\left|f\left(\xi_{i}^{\prime}\right)-f_{n}\left(\xi_{i}^{\prime}\right)\right|\left(t_{i}^{\prime}-t_{i-1}^{\prime}\right)<(b-a) \bigvee_{i=1}^{\infty} a_{i, \varphi(i+2)}
$$

for each $\delta$-fine HK partition $\mathcal{D}_{2}$ and $n \geqslant p_{2}$. We can choose a $(D)$-sequence $\left(c_{i, j}\right)_{i, j}$ of elements of $X$ such that

$$
\bigvee_{i=1}^{\infty} b_{i, \varphi(i+1)}+\bigvee_{i=1}^{\infty} b_{i, \varphi(i+2)}+(b-a) \bigvee_{i=1}^{\infty} a_{i, \varphi(i+1)}+(b-a) \bigvee_{i=1}^{\infty} a_{i, \varphi(i+2)} \leqslant \bigvee_{i=1}^{\infty} c_{i, \varphi(i)}
$$

Let $n>\max \left\{p_{1}, p_{2}\right\}$. Then,

$$
\begin{aligned}
\left|S\left(f, \mathcal{D}_{1}, \delta\right)-S\left(f, \mathcal{D}_{2}, \delta\right)\right|= & \left|S\left(f, \mathcal{D}_{1}, \delta\right)-S\left(f_{n}, \mathcal{D}_{1}, \delta\right)\right|+\left|S\left(f_{n}, \mathcal{D}_{1}, \delta\right)-\int_{a}^{b} f_{n}(t) \Delta t\right| \\
& +\left|\int_{a}^{b} f_{n}(t) \Delta t-S\left(f_{n}, \mathcal{D}_{2}, \delta\right)\right|+\left|S\left(f_{n}, \mathcal{D}_{2}, \delta\right)-S\left(f, \mathcal{D}_{2}, \delta\right)\right|<\bigvee_{i=1}^{\infty} c_{i, \varphi(i)}
\end{aligned}
$$

for each $\delta$-fine $H K$ partition $\mathcal{D}_{1}$ and $\mathcal{D}_{2}$ of $[a, b]_{\mathbb{T}}$. Therefore, by Theorem $3.4, f$ is $H K \Delta$-integrable. 
Step 2. Since $f$ is HK $\Delta$-integrable, there exists a $(D)$-sequence $\left(c_{i, j}\right)_{i, j}$ of elements of $X$ such that for every $\varphi \in \mathbb{N}^{\mathbb{N}}$, there exists a $\Delta$-gauge $\delta^{\prime}$ for $[a, b]_{\mathbb{T}}$, such that

$$
\left|S\left(f, \mathcal{D}^{\prime}, \delta^{\prime}\right)-\int_{a}^{b} f(t) \Delta t\right|<\bigvee_{i=1}^{\infty} c_{i, \varphi(i+1)}
$$

for each $\delta^{\prime}$-fine $H K$ partition $\mathcal{D}^{\prime}$ of $[a, b]_{\mathbb{T}}$. By the uniform HK $\Delta$-integrability, there exists a (D)-sequence $\left(e_{i, j}\right)_{i, j}$ of elements of $X$ for every $\varphi \in \mathbb{N}^{\mathbb{N}}$ such that

$$
\left|S\left(f_{n}, \mathcal{D}^{\prime}, \delta^{\prime}\right)-\int_{a}^{b} f_{n}(t) \Delta t\right|<\bigvee_{i=1}^{\infty} b_{i, \varphi(i+3)}
$$

for each $\delta^{\prime}$-fine $H K$ partition $\mathcal{D}^{\prime}$ of $[a, b]_{\mathbb{T}}$ and $n \in \mathbb{N}$. By the w.c.r.s. convergence,

$$
\left|S\left(f, \mathcal{D}^{\prime}, \delta^{\prime}\right)-S\left(f_{n}, \mathcal{D}^{\prime}, \delta^{\prime}\right)\right|<(b-a) \bigvee_{i=1}^{\infty} a_{i, \varphi(i+3)}
$$

for each $\delta^{\prime}$-fine HK partition $\mathcal{D}^{\prime}$ and $n \geqslant p$. Choose a $(D)$-sequence $\left(d_{i, j}\right)_{i, j}$ of elements of $X$ such that

$$
\bigvee_{i=1}^{\infty} c_{i, \varphi(i+1)}+\bigvee_{i=1}^{\infty} b_{i, \varphi(i+3)}+(b-a) \bigvee_{i=1}^{\infty} a_{i, \varphi(i+3)} \leqslant \bigvee_{i=1}^{\infty} d_{i, \varphi(i)} .
$$

Then,

$$
\begin{aligned}
\left|\int_{a}^{b} f(t) \Delta t-\int_{a}^{b} f_{n}(t) \Delta t\right|= & \left|\int_{a}^{b} f(t) \Delta t-S\left(f, \mathcal{D}^{\prime}, \delta^{\prime}\right)\right|+\left|S\left(f, \mathcal{D}^{\prime}, \delta^{\prime}\right)-S\left(f_{n}, \mathcal{D}^{\prime}, \delta^{\prime}\right)\right| \\
& +\left|S\left(f_{n}, \mathcal{D}^{\prime}, \delta^{\prime}\right)-\int_{a}^{b} f_{n}(t) \Delta t\right|<\bigvee_{i=1}^{\infty} d_{i, \varphi(i)}
\end{aligned}
$$

for each $\delta^{\prime}$-fine $H K$ partition $\mathcal{D}^{\prime}$ of $[a, b]_{\mathbb{T}}$. It follows that

$$
\lim _{n \rightarrow \infty} \int_{a}^{b} f_{n}(t) \Delta t=\int_{a}^{b} f(t) \Delta t
$$

and the proof is complete.

We now recall the well-known Fremlin lemma.

Lemma 4.4 ([29]). Let $\left\{\left(a_{i, j}^{n}\right)_{i, j}: n \in \mathbb{N}\right\}$ be any countable family of regulators. Then, for each fixed element $x \in X$, $x \geqslant 0$, there exists $a(D)$-sequence $\left(a_{i, j}\right)_{i, j}$ of elements of $X$ such that

$$
x \bigwedge \sum_{i=1}^{\infty}\left(\bigvee_{i=1}^{\infty} a_{i, \varphi(i)+n}^{n} \leqslant \bigvee_{i=1}^{\infty} a_{i, \varphi(i)}\right.
$$

for every $\varphi \in \mathbb{N}^{\mathbb{N}}$.

Theorem 4.5 (Monotone convergence theorem). Let $\left\{f_{n}\right\}_{n=1}^{\infty}$ be a sequence of HK $\Delta$-integrable functions on $[a, b]_{\mathbb{T}}$, and $f_{1}$ be bounded from below. Let $f:[a, b]_{\mathbb{T}} \rightarrow X$ be a bounded function such that $f_{n} \leqslant f_{n+1}$ and $f_{n} \rightarrow f$ converges with a common regulating sequence. Then, $\mathrm{f}$ is $\mathrm{HK} \Delta$-integrable and

$$
\lim _{n \rightarrow \infty} \int_{a}^{b} f_{n}(t) \Delta t=\int_{a}^{b} f(t) \Delta t
$$


Proof. Since $f_{n}$ are HK $\Delta$-integrable functions on $[a, b]_{\mathbb{T}}$, there exists a (D)-sequence $\left(a_{n, i, j}\right)_{i, j}$ of elements of $X$ such that for every $\varphi \in \mathbb{N}^{\mathbb{N}}$ there exists a $\Delta$-gauge $\delta_{n}$ for $[a, b]_{\mathbb{T}}$, such that

$$
\left|S\left(f_{n}, \mathcal{D}_{n}, \delta_{n}\right)-\int_{a}^{b} f_{n}(t) \Delta t\right|<\bigvee_{i=1}^{\infty} a_{n, i, \varphi(i+n+1)}
$$

for each $\delta_{n}$-fine HK partition $\mathcal{D}_{\mathfrak{n}}$ of $[a, b]_{\mathbb{T}}$. By the w.c.r.s. convergence, there exists a (D)-sequence $\left(a_{i, j}\right)_{i, j}$ of elements of $X$ such that for every $\varphi \in \mathbb{N}^{\mathbb{N}}$ and every $t \in[a, b]_{\mathbb{T}}$, there exists $p=p(t)$ such that

$$
\left|f_{n}(t)-f(t)\right|<\bigvee_{i=1}^{\infty} a_{i, \varphi(i+1)}
$$

for any $n \geqslant p$. Let $b_{1, i, j}=2(b-a) a_{i, j}, b_{m, i, j}=a_{m-1, i, j}, m=2,3, \ldots$, and $x=(b-a)(L-l)$, where $L, l \in X$ are such that $l \leqslant f_{1}(\xi) \leqslant f(\xi) \leqslant L$ for any $\xi \in[a, b]_{\mathbb{T}}$. By Fremlin's Lemma 4.4, there exists a (D)-sequence $\left(b_{i, j}\right)_{i, j}$ of elements of $X$ such that for every $\varphi \in \mathbb{N}^{\mathbb{N}}$

$$
x \bigwedge\left(\sum_{m=1}^{\infty} \bigvee_{i=1}^{\infty} b_{m, i, \varphi(i+m)}\right) \leqslant \bigvee_{i=1}^{\infty} b_{i, \varphi(i)}
$$

Let $\varphi \in \mathbb{N}^{\mathbb{N}}$ and $\delta(\xi)=\min \left\{\delta_{1}(\xi), \delta_{2}(\xi), \ldots, \delta_{\mathfrak{p}(\mathfrak{t})}(\xi)\right\}, \mathcal{D}^{0}=\mathcal{D}^{1} \cup \mathcal{D}^{2}$, where

$$
\mathcal{D}^{1}=\left\{\left(\left[t_{k-1}, t_{k}\right]_{\mathbb{T}}, \xi_{k}\right) \in \mathcal{D} \mid p\left(\xi_{k}\right) \geqslant n\right\}, \quad \mathcal{D}^{2}=\bigcup_{p\left(\xi_{k}\right)<n} \mathcal{D}_{k},
$$

with $\mathcal{D}_{k}$ a sufficiently fine partition of $\left[t_{k-1}, t_{k}\right]_{\mathbb{T}}$ such that $\mathcal{D}^{0}$ is $\delta_{n}$-fine. Thanks to Henstock's Lemma 3.6, we have

$$
\left|\sum_{p\left(\xi_{k}\right) \geqslant n} f_{n}\left(\xi_{k}\right)\left(t_{k}-t_{k-1}\right)-\sum_{p\left(\xi_{k}\right) \geqslant n} \int_{t_{k-1}}^{t_{k}} f_{n}(t) \Delta t\right| \leqslant \bigvee_{i=1}^{\infty} a_{n, i, \varphi(i+n+1)} .
$$

Consequently, we obtain

$$
\begin{aligned}
& \left|S\left(f_{n}, \mathcal{D}_{n}, \delta\right)-\int_{a}^{b} f_{n}(t) \Delta t\right| \\
& \leqslant\left|\sum_{p\left(\xi_{k}\right) \geqslant n} f_{n}\left(\xi_{k}\right)\left(t_{k}-t_{k-1}\right)-\sum_{p\left(\xi_{k}\right) \geqslant n} \int_{t_{k-1}}^{t_{k}} f_{n}(t) \Delta t\right| \\
& +\left|\sum_{p\left(\xi_{k}\right)<n} f_{n}\left(\xi_{k}\right)\left(t_{k}-t_{k-1}\right)-\sum_{p\left(\xi_{k}\right)<n} \int_{t_{k-1}}^{t_{k}} f_{n}(t) \Delta t\right| \\
& \leqslant \bigvee_{i=1}^{\infty} a_{n, i, \varphi(i+n+1)}+\left|\sum_{m=1}^{n-1} \sum_{p} f_{\left(\xi_{k}\right)=m}\left(\xi_{k}\right)\left(t_{k}-t_{k-1}\right)-\sum_{m=1}^{n-1} \sum_{p\left(\xi_{k}\right)=m} \int_{t_{k-1}}^{t_{k}} f_{n}(t) \Delta t\right| \\
& \leqslant \bigvee_{i=1}^{\infty} a_{n, i, \varphi(i+n+1)}+\left|\sum_{m=1}^{n-1} \sum_{p} f_{\left(\xi_{k}\right)=m}\left(\xi_{k}\right)\left(t_{k}-t_{k-1}\right)-\sum_{m=1}^{n-1} \sum_{p\left(\xi_{k}\right)=m} \int_{t_{k-1}}^{t_{k}} f_{\xi_{k}}(t) \Delta t\right| \\
& \leqslant \bigvee_{i=1}^{\infty} a_{n, i, \varphi(i+n+1)}+\left|\sum_{m=1}^{n-1} \sum_{p\left(\xi_{k}\right)=m} f_{n}\left(\xi_{k}\right)\left(t_{k}-t_{k-1}\right)-\sum_{m=1}^{n-1} \sum_{p\left(\xi_{k}\right)=m} f_{p\left(\xi_{k}\right)}\left(\xi_{k}\right)\left(t_{k}-t_{k-1}\right)\right|
\end{aligned}
$$




$$
\begin{aligned}
& +\left|\sum_{m=1}^{n-1} \sum_{p\left(\xi_{k}\right)=m} f_{p\left(\xi_{k}\right)}\left(\xi_{k}\right)\left(t_{k}-t_{k-1}\right)-\sum_{m=1}^{n-1} \sum_{p\left(\xi_{k}\right)=m} \int_{t_{k-1}}^{t_{k}} f_{\xi_{k}}(t) \Delta t\right| \\
\leqslant & \bigvee_{i=1}^{\infty} a_{n, i, \varphi(i+n+1)}+\sum_{m=1}^{n-1} \sum_{p\left(\xi_{k}\right)=m}\left|f_{n}\left(\xi_{k}\right)-f_{p\left(\xi_{k}\right)}\left(\xi_{k}\right)\right|\left(t_{k}-t_{k-1}\right) \\
& +\sum_{m=1}^{n-1}\left|\sum_{p\left(\xi_{k}\right)=m} f_{p\left(\xi_{k}\right)}\left(\xi_{k}\right)\left(t_{k}-t_{k-1}\right)-\sum_{p\left(\xi_{k}\right)=m} \int_{t_{k-1}}^{t_{k}} f_{\xi_{k}}(t) \Delta t\right| \\
< & \bigvee_{i=1}^{\infty} a_{n, i, \varphi(i+n+1)}+2(b-a) \bigvee_{i=1}^{\infty} a_{i, \varphi(i+1)}+\sum_{m=1}^{\infty} \bigvee_{i=1}^{\infty} a_{m, i, \varphi(i+m+1)} \\
< & \bigvee_{i=1}^{\infty} b_{1, i, \varphi(i+n+1)}+\sum_{m=1}^{n-1} \bigvee_{i=1}^{\infty} a_{m, i, \varphi(i+m+1)} \\
< & \bigvee_{i=1}^{\infty} b_{1, i, \varphi(i+n+1)}+\sum_{m=2}^{n-1} \bigvee_{i=1}^{\infty} b_{m, i, \varphi(i+m+1)} \\
< & \sum_{m=1}^{\infty} \bigvee_{i=1}^{\infty} b_{m, i, \varphi(i+m) .}
\end{aligned}
$$

On the other hand, we have

$$
\left|S\left(f_{n}, \mathcal{D}, \delta\right)-\int_{a}^{b} f_{n}(t) \Delta t\right| \leqslant(L-l)(b-a)=x .
$$

Then,

$$
\left|S\left(f_{n}, \mathcal{D}, \delta\right)-\int_{a}^{b} f_{n}(t) \Delta t\right| \leqslant x \bigwedge\left(\sum_{m=1}^{\infty} \bigvee_{i=1}^{\infty} b_{m, i, \varphi(i+m)}\right) \leqslant \bigvee_{i=1}^{\infty} b_{i, \varphi(i)} .
$$

Now, we prove that $\left\{f_{n}\right\}_{\mathfrak{n}=1}^{\infty}$ is a sequence of uniformly HK $\Delta$-integrable functions on $[a, b]_{\mathbb{T}}$. By Theorem $4.3, \mathrm{f}$ is HK $\Delta$-integrable and

$$
\lim _{n \rightarrow \infty} \int_{a}^{b} f_{n}(t) \Delta t=\int_{a}^{b} f(t) \Delta t
$$

The proof is complete.

\section{Acknowledgment}

This research is supported by Educational Commission of Hubei Province, grant no. B2016160 (You and Zhao) and by FCT and CIDMA within project UID/MAT/04106/2013 (Torres). The authors are grateful to one anonymous referee for valuable comments and suggestions.

\section{References}

[1] G. Antunes Monteiro, A. Slavík, Generalized elementary functions, J. Math. Anal. Appl., 411 (2014), 838-852. 1

[2] G. Antunes Monteiro, A. Slavík, Extremal solutions of measure differential equations, J. Math. Anal. Appl., 444 (2016), 568-597.

[3] S. Avsec, B. Bannish, B. Johnson, S. Meckler, The Henstock-Kurzweil delta integral on unbounded time scales, PanAmer. Math. J., 16 (2006), 77-98. 1

[4] R. G. Bartle, A modern theory of integration, Graduate Studies in Mathematics, American Mathematical Society, Providence, RI, (2001). 1 
[5] N. Benkhettou, A. M. C. Brito da Cruz, D. F. M. Torres, A fractional calculus on arbitrary time scales: fractional differentiation and fractional integration, Signal Process., 107 (2015), 230-237. 1

[6] N. Benkhettou, A. M. C. Brito da Cruz, D. F. M. Torres, Nonsymmetric and symmetric fractional calculi on arbitrary nonempty closed sets, Math. Methods Appl. Sci., 39 (2016), 261-279. 1

[7] A. Boccuto, Differential and integral calculus in Riesz spaces, Real functions, Liptovský Ján, (1996), Tatra Mt. Math. Publ., 14 (1998), 293-323. 1

[8] A. Boccuto, Integration by parts with respect to the Henstock-Stieltjes in Riesz spaces, preprint, University of Perugia, (1999), 18 pages.

[9] A. Boccuto, D. Candeloro, B. Riečan, Abstract generalized Kurzweil-Henstock-type integrals for Riesz space-valued functions, Real Anal. Exchange, 34 (2009), 171-194.

[10] A. Boccuto, D. Candeloro, A. R. Sambucini, A Fubini theorem in Riesz spaces for the Kurzweil-Henstock integral, J. Funct. Spaces Appl., 9 (2011), 283-304.

[11] A. Boccuto, B. Riečan, A note on improper Kurzweil-Henstock integral in Riesz spaces, Acta Math. (Nitra), 5 (2002), $15-24$.

[12] A. Boccuto, B. Riečan, On the Henstock-Kurzweil integral for Riesz-space-valued functions defined on unbounded intervals, Czechoslovak Math. J., 54 (2004), 591-607.

[13] A. Boccuto, B. Riečan, The Kurzweil-Henstock integral for Riesz space-valued maps defined in abstract topological spaces and convergence theorems, PanAmer. Math. J., 16 (2006), 63-79.

[14] A. Boccuto, B. Riečan, M. Vrábelová, Kurzweil-Henstock integral in Riesz spaces, Bentham Science Publishers, United Arab Emirates, (2009).

[15] A. Boccuto, V. A. Skvortsov, Henstock-Kurzweil type integration of Riesz-space-valued functions and applications to Walsh series, Real Anal. Exchange, 29 (2004), 419-438.

[16] A. Boccuto, V. A. Skvortsov, Comparison of some Henstock-type integrals in the class of functions with values in Riesz spaces, (Russian); translated from Vestnik Moskov. Univ. Ser. I Mat. Mekh., 2006 (2006), 13-18, Moscow Univ. Math. Bull., 61 (2006), 12-17.

[17] A. Boccuto, V. A. Skvortsov, On Kurzweil-Henstock type integrals with respect to abstract derivation bases for Rieszspace-valued functions, J. Appl. Funct. Anal., 1 (2006), 251-270.

[18] A. Boccuto, V. A. Skvortsov, F. Tulone, Integration of functions with values in a complex Riesz space and some applications in harmonic analysis, (Russian); translated from Mat. Zametki, 98 (2015), 12-26, Math. Notes, 98 (2015), 25-37. 1

[19] M. Bohner, T.-X. Li, Oscillation of second-order p-Laplace dynamic equations with a nonpositive neutral coefficient, Appl. Math. Lett., 37 (2014), 72-76. 1

[20] M. Bohner, A. Peterson, Dynamic equations on time scales, An introduction with applications, Birkhäuser Boston, Inc., Boston, MA, (2001).

[21] M. Bohner (Ed.), A. Peterson (Ed.), Advances in dynamic equations on time scales, Birkhäuser Boston, Inc., Boston, MA, (2003).

[22] M. J. Bohner, S. H. Saker, Sneak-out principle on time scales, J. Math. Inequal., 10 (2016), 393-403. 1

[23] B. Bongiorno, L. Di Piazza, K. Musiał, Kurzweil-Henstock and Kurzweil-Henstock-Pettis integrability of strongly measurable functions, Math. Bohem., 131 (2006), 211-223. 1

[24] P. S. Bullen, P. Y. Lee, J. L. Mawhin, P. Muldowney, W. F. Pfeffer, New integrals, Proceedings of the Henstock Conference held in Coleraine, Northern Ireland, (1988).

[25] T. S. Chew, On Kurzweil generalized ordinary differential equations, J. Differential Equations, 76 (1988), 286-293. 1

[26] M. Cichoń, On integrals of vector-valued functions on time scales, Commun. Math. Anal., 11 (2011), 94-110. 1

[27] L. Di Piazza, K. Musiał, Relations among Henstock, McShane and Pettis integrals for multifunctions with compact convex values, Monatsh. Math., 173 (2014), 459-470. 1

[28] M. Federson, J. G. Mesquita, A. Slavík, Measure functional differential equations and functional dynamic equations on time scales, J. Differential Equations, 252 (2012), 3816-3847. 1

[29] D. H. Fremlin, A direct proof of the Matthes-Wright integral extension theorem, J. London Math. Soc., 11 (1975), 276284. 4.4

[30] R. A. Gordon, The integrals of Lebesgue, Denjoy, Perron, and Henstock, Graduate Studies in Mathematics, American Mathematical Society, Providence, RI, (1994). 1

[31] G. S. Guseinov, Integration on time scales, J. Math. Anal. Appl., 285 (2003), 107-127. 1

[32] S. Heikkilä, Differential and integral equations with Henstock-Kurzweil integrable functions, J. Math. Anal. Appl., 379 (2011), 171-179. 1

[33] S. Heikkilä, A. Slavík, On summability, multipliability, product integrability, and parallel translation, J. Math. Anal. Appl., 433 (2016), 887-934.

[34] R. Henstock, A Riemann-type integral of Lebesgue power, Canad. J. Math., 20 (1968), 79-87. 1

[35] R. Henstock, Lectures on the theory of integration, Series in Real Analysis, World Scientific Publishing Co., Singapore, (1988). 1

[36] R. Henstock, The general theory of integration, Oxford Mathematical Monographs, Oxford Science Publications, The Clarendon Press, Oxford University Press, New York, (1991). 1

[37] S. Hilger, Ein Maß kettenkalkül mit Anwendung auf Zentrumannigfahigkeiten, Ph.D. Thesis, Universtät Würzburg, (1988). 1 
[38] D. S. Kurtz, C. W. Swartz, Theories of integration, The integrals of Riemann, Lebesgue, Henstock-Kurzweil, and McShane, Second edition, Series in Real Analysis, World Scientific Publishing Co. Pte. Ltd., Hackensack, NJ, (2012). 1

[39] J. Kurzweil, Generalized ordinary differential equations and continuous dependence on a parameter, (Russian) Czechoslovak Math. J., 7 (1957), 418-449. 1

[40] J. Kurzweil, Henstock-Kurzweil integration: its relation to topological vector spaces, Series in Real Analysis, World Scientific Publishing Co., Inc., River Edge, NJ, (2000). 1

[41] J. Kurzweil, Generalized ordinary differential equations, Not absolutely continuous solutions, Series in Real Analysis, World Scientific Publishing Co. Pte. Ltd., Hackensack, NJ, (2012).

[42] S. Leader, The Kurzweil-Henstock integral and its differentials, A unified theory of integration on $\mathbb{R}^{\text {and }} \mathbb{R}^{\mathrm{n}}$. Monographs and Textbooks in Pure and Applied Mathematics, Marcel Dekker, Inc., New York, (2001).

[43] P. Y. Lee, Lanzhou lectures on Henstock integration, Series in Real Analysis, World Scientific Publishing Co., Inc., Teaneck, NJ, (1989).

[44] T. Y. Lee, Henstock-Kurzweil integration on Euclidean spaces, Series in Real Analysis, World Scientific Publishing Co. Pte. Ltd., Hackensack, NJ, (2011).

[45] P. Y. Lee, R. Výborný, Integral: an easy approach after Kurzweil and Henstock, Australian Mathematical Society Lecture Series, Cambridge University Press, Cambridge, (2000). 1

[46] M. D. Ortigueira, D. F. M. Torres, J. J. Trujillo, Exponentials and Laplace transforms on nonuniform time scales, Commun. Nonlinear Sci. Numer. Simul., 39 (2016), 252-270. 1

[47] A. Peterson, B. Thompson, Henstock-Kurzweil delta and nabla integrals, J. Math. Anal. Appl., 323 (2006), 162-178. 1

[48] W. F. Pfeffer, The Riemann approach to integration, Local geometric theory, Cambridge Tracts in Mathematics, Cambridge University Press, Cambridge, (1993). 1

[49] B. Riečan, On the Kurzweil integral for functions with values in ordered spaces, I, Acta Math. Univ. Comenian., 56/57 (1990), 75-83. 1

[50] B. Riečan, T. Neubrunn, Integral, measure, and ordering, Appendix A by Ferdinand Chovanec and František Kôpka, Appendix B by Hana Kirchheimová and Zdenka Riečanová, Mathematics and its Applications, Kluwer Academic Publishers, Dordrecht; Ister Science, Bratislava, (1997).

[51] B. Riečan, M. Vrábelová, On integration with respect to operator valued measures in Riesz spaces, Tatra Mt. Math. Publ., 2 (1993), 149-165.

[52] B. Riečan, M. Vrábelová, On the Kurzweil integral for functions with values in ordered spaces, II, Math. Slovaca, 43 (1993), 471-475.

[53] B. Riečan, M. Vrábelová, The Kurzweil construction of an integral in ordered spaces, Czechoslovak Math. J., 48 (1998), 565-574. 1

[54] B. R. Satco, C. O. Turcu, Henstock-Kurzweil-Pettis integral and weak topologies in nonlinear integral equations on time scales, Math. Slovaca, 63 (2013), 1347-1360. 1

[55] Š. Schwabik, Generalized ordinary differential equations, Series in Real Analysis, World Scientific Publishing Co., Inc., River Edge, NJ, (1992). 1

[56] Š. Schwabik, G.-J. Ye, Topics in Banach space integration, Series in Real Analysis, World Scientific Publishing Co. Pte. Ltd., Hackensack, NJ, (2005). 1

[57] A. Sikorska-Nowak, Integrodifferential equations on time scales with Henstock-Kurzweil-Pettis delta integrals, Abstr. Appl. Anal., 2010 (2010), 17 pages. 1

[58] A. Sikorska-Nowak, Integro-differential equations on time scales with Henstock-Kurzweil delta integrals, Discuss. Math. Differ. Incl. Control Optim., 31 (2011), 71-90.

[59] A. Slavík, Generalized differential equations: differentiability of solutions with respect to initial conditions and parameters, J. Math. Anal. Appl., 402 (2013), 261-274. 1

[60] A. Slavík, Kurzweil and McShane product integration in Banach algebras, J. Math. Anal. Appl., 424 (2015), 748-773. 1

[61] A. Slavík, Well-posedness results for abstract generalized differential equations and measure functional differential equations, J. Differential Equations, 259 (2015), 666-707. 1

[62] B. S. Thomson, Henstock-Kurzweil integrals on time scales, PanAmer. Math. J., 18 (2008), 1-19. 1

[63] M. Vrábelová, B. Riečan, On the Kurzweil integral for functions with values in ordered spaces, III, Real functions, Liptovský Ján, (1994), Tatra Mt. Math. Publ., 8 (1996), 93-100. 1

[64] G.-J. Ye, On Henstock-Kurzweil and McShane integrals of Banach space-valued functions, J. Math. Anal. Appl., 330 (2007), 753-765. 1

[65] J. H. Yoon, On Henstock-Stieltjes integrals of interval-valued functions on time scales, J. Chungcheong Math. Soc., 29 (2016), 109-115. 1

[66] D.-F. Zhao, G.-J. Ye, On AP-Henstock-Stieltjes integral, J. Chungcheong Math. Soc., 19 (2006), 177-187. 1 